\title{
Spectroscopic Influence of Temperature Inhomogeneities
}

\author{
Roger Cayrel \\ Observatoire de Paris, 61 av. de l'Observatoire F-75014 Paris, France
}

Matthias Steffen

Astrophysikalisches Institut Potsdam, Potsdam, Germany

\begin{abstract}
The effect of temperature inhomogeneities on the formation of two oxygen lines and one Fe II line has been investigated based on 2-D radiation hydrodynamics simulations of convection in the atmospheres of metal-poor stars. It was expected that the IR O I triplet lines would be enhanced by the temperature inhomogeneities, more than the forbidden [O I] line or Fe II lines. The actual computations done on two snapshots under the assumption of LTE have not confirmed this expectation, instead suggesting that the main difference between 'classical' and 'dynamical' atmospheres of metal-poor dwarfs is the significantly cooler mean temperature of the dynamical upper photosphere $\left(\tau_{\text {Ross }} \lesssim 0.01\right)$.
\end{abstract}

\section{Introduction}

The oxygen abundance ${ }^{1}$ in stars can be determined from several spectral features: the permitted triplet of O I near $777 \mathrm{~nm}$, the forbidden line [O I] at $630 \mathrm{~nm}$, or molecular bands of OH, either in the UV near $320 \mathrm{~nm}$, or in the IR near $1556 \mathrm{~nm}$. A long-standing puzzle to be resolved is that these different features give frequently discordant values for the oxygen abundance (see e.g. Israelian et al. 1998, Fulbright \& Kraft 1999 and the talk by R. Kraft at JD8). Roughly speaking, the forbidden line agrees with the IR OH, and the O I triplet agrees with OH UV (Boesgaard et al. 1999). King (1993) found that raising the zero point of the effective temperature scale by 150 to $200 \mathrm{~K}$ considerably reduces the $[\mathrm{O} \mathrm{I}]$ line versus triplet lines discrepancy.

In this paper we investigate 2-D effects on the IR triplet and on the [O I] line, as well as on a weak $\mathrm{Fe}$ II line, often used for determining the $\mathrm{O} / \mathrm{Fe}$ ratio (Nissen \& Edvardsson 1992). It can be presumed that the IR triplet, which has a very high excitation potential $(9.15 \mathrm{eV})$, (i) originates from deep layers where convection is well developed, and (ii) is highly sensitive to temperature fluctuations. For these reasons we considered useful a detailed investigation of the spectroscopic effects of temperature inhomogeneities in the atmospheres of metal-poor stars.

\footnotetext{
${ }^{1} \mathrm{R}$. Cayrel reported a VLT measurement of $[\mathrm{O} / \mathrm{Fe}]=0.7 \pm 0.25$ in $\mathrm{BD}+233130$ in his talk. More details in the NARev JD8 issue
} 


\section{A simplistic analytical argument}

If the equivalent width of a weak line $W$ depends exponentially on the reciprocal of the temperature $\theta=5040 / T$, as suggested by the form of the Saha-Boltzmann equations,

$$
W=W_{0} \exp (-\alpha \Delta \theta)
$$

where $\Delta \theta=\theta-\theta_{0}$, and if $\theta$ is a random variable normally distributed with mean $\theta_{0}$ and standard deviation $\sigma$, then it can be shown by analytical integration that:

$$
\langle W\rangle=W_{0} \exp \left(\frac{1}{2}(\alpha \sigma)^{2}\right)
$$

i.e. that the mean value $\langle W\rangle$ of the equivalent width formed in a perturbed medium is always greater than the equivalent width for the unperturbed medium. If $\alpha \sigma$ is small enough, the effect is negligible, but if e.g. $\sigma=0.08$ and $\alpha \simeq 15$, the effect is very substantial - more than a factor of 2 . For $T_{\text {eff }}=5800 \mathrm{~K}$ these values correspond to $10 \%$ temperature fluctuations and $6.5 \mathrm{eV}$ excitation potential. In reality, however, the line formation process is much more complex.

\section{Actual computations}

We have used for our numerical analysis two snapshots from 2-D hydrodynamical simulations computed previously by one of us (M.S.) for the investigation of multidimensional effects on the lithium resonance line, including NLTE effects (Cayrel \& Steffen 1999). This time we have not yet included NLTE effects, because it was more difficult than for lithium, a trace element. The simulations are for an atmosphere having the effective temperature and the surface gravity of the Sun, but a 100 times smaller metallicity. Fig. 1 shows the response of three lines to the convective temperature fluctuations. The strength of the O I $777.4 \mathrm{~nm}$ triplet line varies by more than a factor of 4 (left panel), while the forbidden line [O I] at $630 \mathrm{~nm}$ and the weak Fe II line at $457.6 \mathrm{~nm}$, often used as reference when computing the $\mathrm{O} / \mathrm{Fe}$ ratio, both show a smaller amplitude (right panel). Evaluation of another snapshot gives very similar results.

\begin{tabular}{|c|c|c|c|}
\hline & O I 777.4 & {$[\mathrm{O} \mathrm{I}] 630.0$} & Fe II 457.6 \\
\hline $1-D$ & 5.53 & 0.69 & 11.3 \\
\hline 2-D & 4.85 & 0.96 & 8.5 \\
\hline
\end{tabular}

Table 1. Entries are the EW of lines, $\langle W\rangle(\mathrm{m} \AA)$, in case of 2-D models

A bad surprise came when we compared the mean equivalent widths with those of 1-D mixing-length models computed with the same background physics (EOS, opacity) for the same $T_{\text {eff }}, \log g,[\mathrm{Fe} / \mathrm{H}]$ (Table 1). Everything was going the wrong way. The $\mathrm{O}$ I triplet, instead of becoming stronger in the 2-D model, is weaker by -0.06 dex, and the [O I] line, instead of being unaffected, is becoming about 0.15 dex stronger. So, instead of bringing the derived aburdances closer together, the 2-D LTE results make them more divergent. A closer inspection 

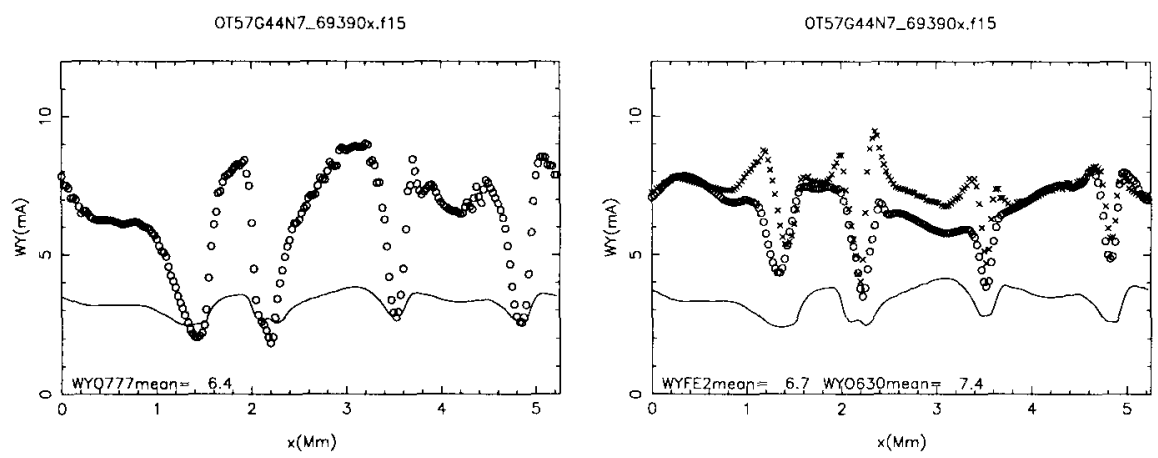

Figure 1. Left: variation of of the O I 777.4 EW along the horizontal coordinate $x$ at disk center (o). Right: behavior of the [O I] 630 line (scaled by a factor of $10, o$ ) and of a weak Fe II line $(+$ ). As expected, these latter two lines have a very similar behavior. In both panels, the continuous line indicates the local continuum intensity.

of the data show that the fluctuations are not the primary cause of the 1-D/2-D differences.

It is the mean temperature of the upper photosphere ( $\tau_{\text {Ross }} \lesssim 0.01$ ), which is significantly cooler in the hydrodynamical models, that is mostly responsible for the differences in line strength. Of course a NLTE treatment may deeply modify these results, possibly decreasing the 1-D/2-D difference, as in the case of lithium. As already shown by Kiselman (1993) and Kiselman and Nordlund (1995), NLTE is important for the O I triplet.

\section{Conclusion}

The conclusion of this preliminary study is that, as such, temperature inhomogeneities do not help to understand the well-known discrepancy between the oxygen abundance derived from the [O I] line and the IR triplet, respectively. The role of NLTE needs further investigation, and the reality of the cool upper photosphere found in the hydrodynamical simulations needs to be confirmed.

\section{References}

Boesgaard, A.M., King, J.R., Delyiannis, C.P., Vogt, S.S. 1999, AJ, 117, 492

Cayrel, R. \& Steffen, M. 1999, IAU Symp. 198, astro-ph/000375

Fulbright, J.P., Kraft, R.P. 1999, ApJ, 118, 527

Israelian G., García López R.J., Rebolo, R. 1998, ApJ, 507, 805

King, J.R. 1993, AJ, 106, 1206

Kiselman, D. 1993, A\&A, 275, 269

Kiselman, D., Nordlund, A. 1995, A\&A, 302, 578

Nissen, P.E., Edvardsson, B. 1992, A\&A, 261, 255 